\title{
Autophagy regulates the degeneration of the auditory cortex through the AMPK-mTOR-ULK1 signaling pathway
}

\author{
JIE YUAN $^{1 *}$, XUEYAN ZHAO $^{1 *}$, YUJUAN HU $^{1 *}$, HAIYING SUN $^{1}$, GUOQING GONG $^{2}$, \\ XIANG HUANG ${ }^{3}$, XUBO CHEN ${ }^{1}$, MINGYU XIA ${ }^{1}$, CHEN SUN $^{1}$, QILIN HUANG ${ }^{1}$, \\ YU SUN ${ }^{1}, \mathrm{WEN} \mathrm{KONG}^{4}$ and WEIJIA KONG ${ }^{1,3}$ \\ ${ }^{1}$ Department of Otolaryngology, Union Hospital, Tongji Medical College, Huazhong University of Science and Technology, \\ Wuhan, Hubei 430022; ${ }^{2}$ Department of Otolaryngology, Central Hospital of Huangshi, Huangshi, Hubei 435000; \\ ${ }^{3}$ Institute of Otorhinolaryngology and ${ }^{4}$ Department of Endocrinology, Union Hospital, Tongji Medical College, \\ Huazhong University of Science and Technology, Wuhan, Hubei 430022, P.R. China
}

Received February 1, 2017; Accepted January 10, 2018

DOI: $10.3892 /$ ijmm.2018.3393

\begin{abstract}
Presbycusis is the most common sensory impairment associated with aging; however, the underlying molecular mechanism remains unclear. Autophagy has been demonstrated to serve a key role in diverse diseases; however, no studies have examined its function in central presbycusis. The aim of the present study was to investigate the changes of autophagy in the physiological processes of the auditory cortex and its role in the degeneration of the auditory cortex, as well as the related mechanisms using naturally aging rats and a D-galactose (D-gal)-induced mimetic rat model of aging. The present study demonstrated that autophagy increased from 3 months to 15 months in the normal saline (NS) control group, while it decreased in the D-gal group. Compared with the age-matched NS group, the D-gal group demonstrated significantly increased levels of the autophagy-related proteins, LC3 and Beclin 1 (BECN1) and the anti-apoptotic proteins B-cell lymphoma (BCL)2 and BCL-extra large (BCL-xL) at 3 months, with no obvious changes in cell apoptosis level and neuron ultrastructural morphology. However, LC3, BECN1,
\end{abstract}

Correspondence to: Professor Wen Kong, Department of Endocrinology, Union Hospital, Tongji Medical College, Huazhong University of Science and Technology, 1277 Jiefang Avenue, Wuhan, Hubei 430022, P.R. China

E-mail:wenly-kong@163.com

Professor Weijia Kong, Department of Otolaryngology, Union Hospital, Tongji Medical College, Huazhong University of Science and Technology, 1277 Jiefang Avenue Wuhan, Hubei 430022, P.R. China

E-mail: entwjkong@hust.edu.cn

*Contributed equally

Key words: autophagy, apoptosis, 5' AMP-activated protein kinase-mechanistic target of rapamycin-ULK1 signaling pathway, auditory cortex, central presbycusis
BCL2 and BCL-xL were decreased at 15 months in the D-gal group, with cell apoptosis significantly increased and substantial neuron degeneration. Additionally, 5' AMP-activated protein kinase (AMPK) activity was enhanced, and mechanistic target of rapamycin (mTOR) and ULK1 phosphorylation (Ser 757) activities were inhibited at 3 months compared with those of the NS group, while the opposite was observed at 9 and 15 months. The present results suggested that autophagy increases from young to adult and decreases at old age in the physiological processes of the auditory cortex, and has anti-apoptotic as well as anti-aging functions in the degeneration of the auditory cortex. Additionally, autophagy was regulated through AMPK activation and mTOR suppression, and impairment of autophagy may serve a key role in the degeneration of the auditory cortex, even in the pathogenesis of central presbycusis.

\section{Introduction}

Age-related hearing loss (ARHL), also known as presbycusis, is a complex degenerative disease characterized by declining auditory function, including increased hearing thresholds and reduced frequency resolution (1). ARHL is one of the most prevalent and chronic conditions of older populations, affecting tens of millions of people worldwide (2), and may cause social isolation, depression, and even dementia (3). In addition to degeneration of the peripheral auditory organs, auditory cortex degeneration has been demonstrated to serve a crucial role in ARHL pathogenesis $(4,5)$; however, the molecular mechanism is not well understood.

D-galactose (D-gal) is a reducing sugar and is oxidized into aldehydes and $\mathrm{H}_{2} \mathrm{O}_{2}$ when present at high levels (6). Chronic systemic exposure of rats to D-gal has been used as an aging model for many years and is widely used to study atherosclerosis (7), reproductive system diseases $(8,9)$ and neurodegenerative diseases, including Alzheimer's disease (10) and ARHL $(11,12)$.

Autophagy is a ubiquitous process that occurs in plant, animal, and fungal cells (13), and three types of autophagy have been described: Macroautophagy (hereafter referred 
to as autophagy), microautophagy and chaperone-mediated autophagy (14). In mammals, autophagy maintains homeostasis by regulating the degradation of cellular organelles and macromolecules and may be activated by starvation, hypoxia and exposure to toxic agents $(13,15-18)$. ULK1 is a serine/threonine kinase, a homologue of yeast autophagy-related protein (Atg)1. In combination with Atg13, FIP200 and Atg101, ULK1 initiates the autophagy process (19). Notably, ULK1 may be phosphorylated by mechanistic target of rapamycin (mTOR) and 5' AMP-activated protein kinase (AMPK) at different sites and perform dual functions (20). mTOR is a well-known negative regulator of autophagy, it phosphorylates ULK1 at Ser 757 when nutrient levels are sufficient and represses its protein kinase activity, thereby blocking the initiation of autophagy (21). However, in response to nutrient deprivation, AMPK is activated and stimulates autophagy by directly activating ULK1 via phosphorylation of Ser 317 and Ser 777 (20). In addition, AMPK promotes activation of autophagy by inhibiting the activity of mTOR complex 1 through phosphorylation of either Raptor or tuberous sclerosis complex 2, which subsequently suppresses the activity of mTOR $(22,23)$. In addition to changes in nutrient levels, AMPK and mTOR may be activated by oxidative stress $(24,25)$, and studies have indicated that AMPK and mTOR are essential modulators of the aging process $(26,27)$. Additionally, previous studies have indicated that AMPK- and mTOR-dependent autophagy is involved in neurodegenerative diseases (28-30). Therefore, it was hypothesized that AMPK and mTOR are involved in the regulation of autophagy in the degeneration of the auditory cortex in the present model. Furthermore, Tsuchihashi et al (25) suggested that 4E binding protein 1 (4EBP1), a key substrate of mTOR, impairs autophagy independent of mTOR, leading to premature senescence in auditory cells (25). However, there were no further studies on this, and whether 4EBP1 regulates autophagy in the degeneration of the auditory cortex is unknown. Thus, this issue was investigated in the present study.

Autophagy has been demonstrated to serve important roles in various diseases, including cancer, heart disease, neurodegeneration, autoimmune diseases, aging and infection (31-35). At the organismal and cellular levels, autophagy has pro-death or pro-survival functions and has different interactions with apoptosis depending on the context (36-38). Nevertheless, to the best of our knowledge, no studies have focused on the role of autophagy and its interactions with apoptosis in central presbycusis.

Changes of autophagy in the physiological/pathological processes of the auditory cortex are unclear, and the role of autophagy, as well as its related mechanisms, in the degeneration of the auditory cortex has not been studied. Therefore, the present study assessed changes of autophagy in the auditory cortex of naturally aging rats and mimetic aging rats by 8 weeks of D-gal injection, and further investigated the role of autophagy and its related mechanism in the degeneration of the auditory cortex.

\section{Materials and methods}

Ethics statement. All animal procedures were performed in strict accordance with the recommendations of the Guide for the Care and Use of Laboratory Animals of the National Institutes of Health (39). The experimental procedures were performed with the approval of the Committee on the Ethics of Animal Care and Use of Huazhong University of Science and Technology (Wuhan, China; permit no. IACUC S512).

Animal procedures. A total of 180 male Sprague Dawley (SD) rats (70.10 $\pm 11.46 \mathrm{~g}, 3$-weeks old) were obtained from the Experimental Animal Centre of Tongji Medical College, Huazhong University of Science and Technology. The rats were individually maintained in a temperature-controlled $\left(23 \pm 2^{\circ} \mathrm{C}\right.$ with $50-60 \%$ relative humidity) room with a $12-\mathrm{h}$ light/dark cycle and free access to water and food. Prior to treatment, the rats were allowed to acclimate for 1 week and were then randomly divided into a control group $(n=90)$ and a mimetic aging group $(n=90)$. The rats in the mimetic aging group were treated with D-gal (500 mg/kg/day; Sigma-Aldrich; Merck KGaA, Darmstadt, Germany) via subcutaneous injection for 8 weeks. The rats in the control group were injected with normal saline (NS; $500 \mathrm{mg} / \mathrm{kg} /$ day) on the same schedule. After the 8-week injection protocol, the rats in the two groups were further divided into a 3-month-old subgroup (just after the last injection), a 9-month-old subgroup (6 months after the last injection), and a 15-month-old subgroup (12 months after the last injection), in which the rats were sacrificed at 3,9 and 15 months, respectively.

DNA extraction and quantification of the mitochondrial (mt) DNA 4,834-bp deletion. Total DNA of the auditory cortex was extracted using a Genomic DNA Purification kit (Tiangen Biotech Co., Ltd., Beijing, China), and TaqMan (Tiangen Biotech Co., Ltd.) quantitative polymerase chain reaction (qPCR) analysis was used to determine the proportion of the mtDNA common deletion (CD). The D-Loop region copy was used as the conservative segment. The primers and probes for the mtDNA CD and mtDNA D-loop are listed in Table I, as previously described (40). PCR amplification was performed using a LC-480 real-time PCR system (Roche Diagnostics, Basel, Switzerland) in a $20-\mu 1$ reaction volume. The cycling conditions included an initial phase at $95^{\circ} \mathrm{C}$ for $30 \mathrm{sec}$, followed by 40 cycles at $95^{\circ} \mathrm{C}$ for $5 \mathrm{sec}$ and at $60^{\circ} \mathrm{C}$ for $30 \mathrm{sec} . \Delta \mathrm{Cq}$ $\left(\mathrm{Cq}_{\text {deletion }}-\mathrm{Cq}_{\mathrm{D} \text {-loop }}\right)$ was used to reflect the abundance of the mtDNA 4,834-bp deletion. The relative expression indicating the factorial difference in the deletions between the NS group and the D-gal-treated group was calculated using the $2^{-\Delta \Delta \mathrm{Cq}}$ method (41), where $\Delta \Delta \mathrm{Cq}=\Delta \mathrm{Cq}_{\mathrm{mtDNA} \text { deletion in D-gal-treated group }}{ }^{-}$

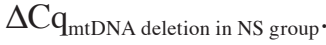

Transmission electron microscopy (TEM). Following deep anesthesia, a total of 36 rats ( $n=6 /$ subgroup) were perfused transcardially with $2.5 \%$ glutaraldehyde subsequent to brief perfusion with $0.9 \%$ sodium chloride. Following perfusion, the brain was dissected from the skull, and the auditory cortex was separated from the brain. Subsequently, the auditory cortex was fixed with the $2.5 \%$ glutaraldehyde at $4^{\circ} \mathrm{C}$ for $12 \mathrm{~h}$, and further fixation was performed using $1 \%$ osmium tetroxide at $4^{\circ} \mathrm{C}$. After $2 \mathrm{~h}$, the auditory cortex was dehydrated using a series of ascending graded ethanol $(50,70,80,85,90,95$ and $100 \%)$. Next, the auditory cortex was treated with propylene oxide for $\sim 0.5 \mathrm{~h}$ at room temperature and then embedded in 
Table I. Probes and primers used for quantitative polymerase chain reaction analysis of mtDNA.

\begin{tabular}{lll}
\hline Name & Probe/primer direction & Sequence $\left(5^{\prime}-3^{\prime}\right)$ \\
\hline D-Loop & Probe & TTGGTTCATCGTCCATACGTTCCCCTTA \\
& Forward primer & GGTTCTTACTTCAGGGCCATCA \\
Reverse primer & GATTAGACCCGTTACCATCGAGAT \\
& Probe & TCACTTTAATCGCCACATCCATAACTGCTGT \\
& Forward primer & GATTAGACCCGTTACCATCGAGAT \\
& Reverse primer & CGAAGTAGATGATCCGTATGCTGTA \\
\hline
\end{tabular}

Table II. Primers used for reverse transcription-quantitative polymerase chain reaction analysis.

\begin{tabular}{lll}
\hline Genes & \multicolumn{1}{c}{ Primer sequence (5'-3') } & Accession no. \\
\hline LC3 & F: GTTAAGCCCCTACCAAGGCA & NM_022867.2 \\
BECN1 & R: AGGGACTGTTTCCAGGGACT & NM_001034117.1 \\
& F: GAATGGAGGGGTCTAAGGCG & N: CTTCCTCCTGGCTCTCTCCT 017059.2 \\
BCL2-associated X protein & F: GGGCCTTTTTGCTACAGGGT & NM_031535.2 \\
BCL-extra large & R: TTCTTGGTGGATGCGTCCTG & FM_016993.1 \\
BCL2 & F: ATCTTGGCTTTGGATCCTGG & R: GGGGCTTCAGTCCTGTTCTC \\
3-actin & F: TCGCGACTTTGCAGAGATGT & R: CAATCCTCCCCCAGTTCACC \\
& F: GCAGGAGTACGATGAGTCCG & NM_031144.3 \\
\hline
\end{tabular}

F, forward; R, reverse; BCL, B-cell lymphoma.

graded araldite (A3183; Sigma-Aldrich; Merck KGaA; 1/3 and $1 / 2$, pure araldite) for $12 \mathrm{~h}$ per step at $37^{\circ} \mathrm{C}$. Following this, the embedded samples were placed in a $60^{\circ} \mathrm{C}$ incubator for $48 \mathrm{~h}$. Serial ultrathin sections $(60-100 \mathrm{~nm})$ were collected on copper grids and stained at room temperature with uranyl acetate for $30 \mathrm{~min}$ and then lead citrate for $10 \mathrm{~min}$. A Tecnai G220 TWIN (FEI; Thermo Fisher Scientific, Inc., Waltham, MA, USA) transmission electron microscope was used to observe the ultrastructure of the sections at x1,700 and x5,000 magnification.

Terminal deoxynucleotidyl-transferase-mediated dUTP nick end labelling (TUNEL) staining. Apoptosis was detected using TUNEL staining. Following deep anesthesia, a total of 36 rats ( $n=6 /$ subgroup) were transcardially perfused with $0.9 \%$ sodium chloride and $4 \%$ paraformaldehyde solution. Following the perfusion, separated brain sections were fixed with $4 \%$ paraformaldehyde solution overnight at $4^{\circ} \mathrm{C}$. Following rinsing with distilled water, brain sections were dehydrated in a graded alcohol series $(70,80,90,95,100,100$ and $100 \%$ ), and immersed in paraffin after clearing in xylene. Serial 5-mm coronal sections containing the auditory cortex were deparaffinized in xylene and rehydrated through graded concentrations of ethanol (100, 100, 95, 90, 80 and 70\%). Following a $0.5-\mathrm{h}$ incubation with proteinase $\mathrm{K}(20 \mu \mathrm{g} / \mathrm{ml}$; Roche Diagnostics) at $37^{\circ} \mathrm{C}$, the sections were stained using an In Situ Cell Death Detection kit (Roche Diagnostics), according to the ratio 1:9 of solution $\mathrm{A}$ and $\mathrm{B}$, and incubated at $37^{\circ} \mathrm{C}$ for $1 \mathrm{~h}$. A DAPI staining solution $(1 \mu \mathrm{g} / \mathrm{ml}$; Beyotime Institute of Biotechnology, Haimen, China) was employed to counterstain the nuclei at room temperature for $10 \mathrm{~min}$. The labelled cells were observed using a laser scanning confocal microscope (Nikon Corporation, Tokyo, Japan), and six fields of view for each section were observed.

$R N A$ extraction and reverse transcription $(R T)-q P C R$ analysis. The auditory cortex tissues from 6 rats in each subgroup were separated and prepared for RNA extraction using TRIzol (Invitrogen; Thermo Fisher Scientific, Inc.). Following this, an aliquot of the extracted RNA was immediately reverse transcribed to cDNA using a PrimeScript RT Reagent kit (Takara Bio, Inc., Otsu, Japan). qPCR was performed with a LightCycler 480 RT-PCR system (Roche Diagnostics) using SYBR-Green II PCR Master Mix (Takara Bio, Inc.). The primers were designed by Takara Bio, Inc., and are listed in Table II. $\beta$-actin was included as an endogenous 
control. The amplification conditions were as follows: $95^{\circ} \mathrm{C}$ for $5 \mathrm{~min} ; 45$ cycles of $95^{\circ} \mathrm{C}$ for $10 \mathrm{sec}, 60^{\circ} \mathrm{C}$ for $20 \mathrm{sec}$, and $72^{\circ} \mathrm{C}$ for $20 \mathrm{sec}$; followed by $95^{\circ} \mathrm{C}$ for $5 \mathrm{sec}$ and $65^{\circ} \mathrm{C}$ for $60 \mathrm{sec}$. The relative mRNA expression levels in the control and mimetic aging groups were calculated using the $2^{-\Delta \Delta \mathrm{Cq}}$ method.

Protein extraction and western blotting. The protein expression levels were examined using western blotting. A total of 36 rats ( $n=6 /$ subgroup) were sacrificed, and their auditory cortices were dissected and lysed in radioimmunoprecipitation assay lysis solution (Beyotime Institute of Biotechnology) containing phosphatase inhibitors and PMSF. A BCA Protein Assay kit (Beyotime Institute of Biotechnology) was used to determine the protein concentrations. The proteins were loaded onto 6 and 12\% SDS-PAGE, separated, and transferred to polyvinylidene difluoride membranes (EMD Millipore, Billerica, MA, USA). The membranes were incubated in blocking solution [5\% non-fat milk (BD Biosciences, Franklin Lakes, NJ, USA) in Tris-buffered saline] for $1 \mathrm{~h}$ at room temperature. Subsequently, the membranes were incubated overnight at $4^{\circ} \mathrm{C}$ with the following primary antibodies: LC3B (ab192890; 1:1,000; Abcam, Cambridge, MA, USA), Beclin 1 (BECN1; ab217179; 1:1,000; Abcam), p62 (ab155686; 1:1,000; Abcam), cleaved caspase 3 (CASP3; 9664; 1:500; Cell Signaling Technology, Inc., Danvers, MA, USA), B-cell lymphoma 2 (BCL2)-associated X protein (BAX; 34260; 1:500; Signalway Antibody LLC, College Park, MD, USA), BCL-extra large (BCL-xL; 21061; 1:500; Signalway Antibody LLC), BCL2 (ab59348; 1:500; Abcam), phosphorylated (p)-AMPK (sc-33524; 1:1,000, Santa Cruz Biotechnology, Inc., Dallas, TX, USA), p-mTOR (SAB4504476; 1:800; Sigma-Aldrich; Merck KGaA), p-ULK1 (14202; 1:1,000; Cell Signaling Technology, Inc.), Atg13 (ab201467; 1:1,000; Abcam), Atg5 (44254; 1:2,000; Signalway Antibody LLC), Atg7 (38148; 1:500; Signalway Antibody LLC), p-4EBP1 (2855; 1:800; Cell Signaling Technology, Inc.) and $\beta$-actin (ab8227; 1:3,000; Abcam). The membranes were incubated with the corresponding secondary antibodies (ANT019; ANT020; 1:4,000; AntGene Biotech Co., Ltd., Wuhan, China) for $60 \mathrm{~min}$ at room temperature. Enhanced chemiluminescent Plus (Beyotime Institute of Biotechnology) was used to visualize the membranes. The protein levels were normalized to the level of $\beta$-actin in the corresponding lanes and ImageJ 10.0 software (National Institutes of Health, Bethesda, MD, USA) was used for densitometry.

Immunofluorescence. The cleaved CASP3, LC3, BECN1, p62 and BCL2 protein levels were also examined using immunofluorescence. Following deep anesthesia, a total of 36 rats ( $n=6 /$ subgroup) were transcardially perfused, and separated brain sections were fixed with $4 \%$ paraformaldehyde solution overnight at $4^{\circ} \mathrm{C}$. Subsequent to rinsing with distilled water, brain sections were dehydrated in graded alcohol series (70, $80,90,95,100,100$ and 100\%), and immersed in paraffin after clearing in xylene. A 5-um paraffin section was deparaffinized in xylene and rehydrated through graded concentrations of ethanol (100, 100, 95, 90, 80 and 70\%). Following 10-min citrate solution antigen repair in boiling water, donkey serum albumin (ANT051; AntGene Biotech Co., Ltd.) was used to block nonspecific binding for $30 \mathrm{~min}$ at room temperature.
Subsequently, the sections were incubated overnight at $4^{\circ} \mathrm{C}$ with the aforementioned primary antibodies diluted to 1:200. The sections were then incubated with a secondary antibody (ANT024; 1:200; AntGene Biotech Co., Ltd.) for $2 \mathrm{~h}$ after repeated washes with Tris-buffered saline-Tween-20. Sections that were not incubated with a primary antibody were used as the negative controls. A laser scanning confocal microscope (Nikon Corporation) was used to observe the staining at $\mathrm{x} 200$ and x600 magnifications.

Statistical analyses. The data were presented as the mean \pm standard error of the mean. The statistical analyses were performed using SPSS 20.0 software (IBM Corp., Armonk, NY, USA). Statistically significant differences between the groups were determined using one-way analysis of variance followed by Tukey's post hoc test, and $\mathrm{P}<0.05$ was considered to indicate a statistically significant difference.

\section{Results}

D-gal treatment elevates mtDNA CD in the auditory cortex. CDs are also called mt 4,834-bp deletions and correspond to mtDNA 4,977-bp deletions in humans (42). Previous research has indicated an association between CD levels and presbycusis (43). TaqMan qPCR analysis demonstrated that D-gal treatment induced significantly higher CD levels in the D-gal groups at all ages compared with the levels in the age-matched NS groups (Fig. 1). In addition, the CDs accumulated with age in both the natural aging group and the mimetic aging group.

D-gal treatment accelerates neurodegeneration and activates apoptosis. Neurons in the auditory cortex in the NS group did not display obvious ultrastructural changes in the 3- and 9-month-old rats (Fig. 2A and B). However, in the 15-month-old rats in the NS group, swollen mitochondria, disrupted myelin and lipofuscin were observed (Fig. 2C). In the D-gal-treated groups, normal nuclei, mitochondria, Golgi apparatus and rough endoplasmic reticulum were observed in the 3-month-old subgroup (Fig. 2D), whereas in the 9-month-old D-gal-treated rats, irregular nuclei, condensed chromatin, accumulated lipofuscin, swollen mitochondria and disrupted myelin were identified (Fig. 2E). Additionally, these changes were more pronounced in the 15-month-old D-gal-treated rats (Fig. 2F). Furthermore, more autophagosomes and auto-lysosomes were observed in the 15-month-old NS group and the 3- and 9-month-old D-gal subgroups, while few autophagosome and auto-lysosomes were identified in the 3- and 9-month-old NS subgroups and 15-month-old D-gal subgroup, particularly in the 15-month-old D-gal subgroup.

The detected protein level of cleaved CASP3 by western blotting and immunofluorescence assays, as well as the results of TUNEL staining (Fig. 3), revealed that apoptosis levels were significantly increased in the 9- and 15-month-old D-gal groups compared with the levels in the respective NS control groups, and this level increased with age in both groups.

D-gal treatment enhances autophagy activity and p62 degradation. The mRNA levels of LC3 and BECN1 were increased at 3 and 9 months in the D-gal-treated groups; however, these levels were decreased at 15 months compared 


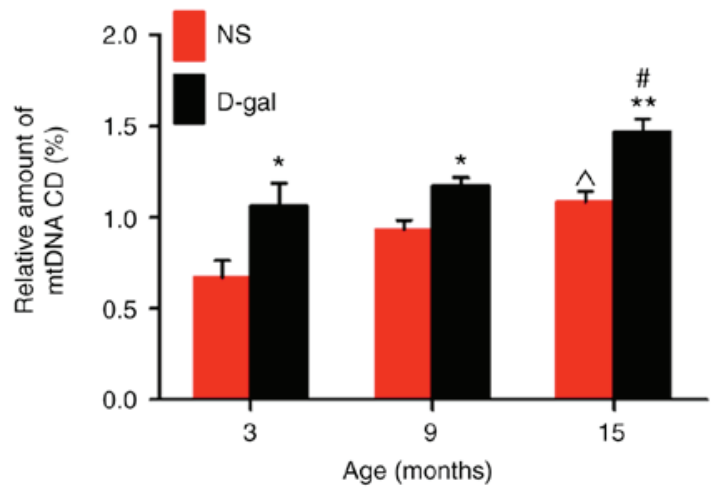

Figure 1. CD levels increase in D-gal-induced mimetic aging rats. A quantitative polymerase chain reaction analysis was used to measure the accumulation of mtDNA CD. The CD levels in the D-gal-induced mimetic aging rats were higher than those in the age-matched NS subgroups, and the CD levels increased with aging in both groups. Data are expressed as mean + standard error of the mean. ${ }^{*} \mathrm{P}<0.05$ and ${ }^{* *} \mathrm{P}<0.01$ vs. the age-matched NS subgroups; ${ }^{\wedge} \mathrm{P}<0.01$ vs. the 3 -month-old NS subgroup; ${ }^{~} \mathrm{P}<0.05$ vs. the 3 -month-old $\mathrm{D}$-gal group. CD, common deletion; D-gal, D-galactose; mtDNA, mitochondrial DNA; NS, normal saline.

with those in the age-matched NS control groups (Fig. 4A a-c). The same trend was observed for the protein expression levels of LC3 and BECN1 (Fig. 4A d-f). The immunofluorescence images demonstrating the protein levels of LC3 and BECN1 in the auditory cortex with aging are shown in Fig. 4B. It was demonstrated that autophagy was increased from 3 months to 15 months in the NS group, while it decreased with aging in the D-gal group.
Furthermore, in addition to $\mathrm{LC} 3$ and BECN1, p62 protein expression levels were detected using western blotting and immunofluorescence assays. p62 expression levels were significantly decreased in the 3-month-old D-gal subgroup compared with the levels in the age-matched NS group, while these levels were significantly increased at 15 months compared with those in the NS groups (Fig. 5). Additionally, these autophagy-related proteins demonstrated approximately equivalent levels in the 9-month-old D-gal induced mimetic aging group and 15-month-old NS group.

Apoptosis and autophagy levels in the auditory cortex change with aging. By investigating the levels of $\mathrm{CD}$, and cell apoptosis and neuronal degeneration at 3,9 and 15 months in the two groups, the present results demonstrated that the aging level in the 15-month-old rats in the NS group was higher than those in 9-month-old NS subgroup. Additionally, the present results indicated that 9-month-old rats in the D-gal-treated group presented an approximately similar life status compared with that of the 15-month-old rats in NS group, which was further confirmed by immunofluorescence analyses of LC3 and BECN1. Furthermore, neuronal degeneration and cell apoptosis were more apparent in the 15-month-old D-gal subgroup than the 9-month-old D-gal subgroup. The present results revealed that the function of D-gal exposure that accelerated the aging in rats was confirmed in the present study, which is consistent with previously research $(6,44)$. The lifespan of the majority of SD rats is $2.5-3$ years, and 15 months is equivalent to the late adult period of rats and roughly equivalent to 40-year-old humans (45). In other words,
A
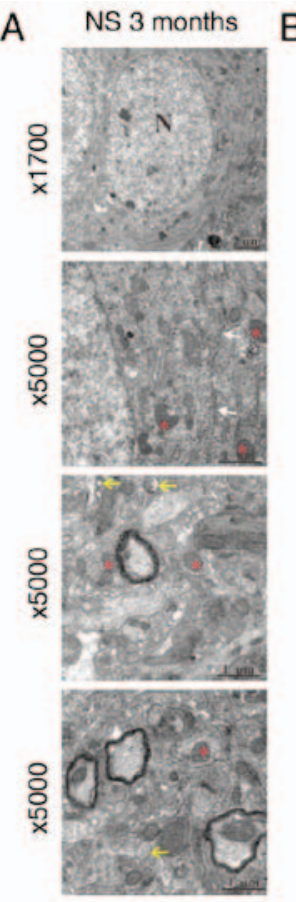

B
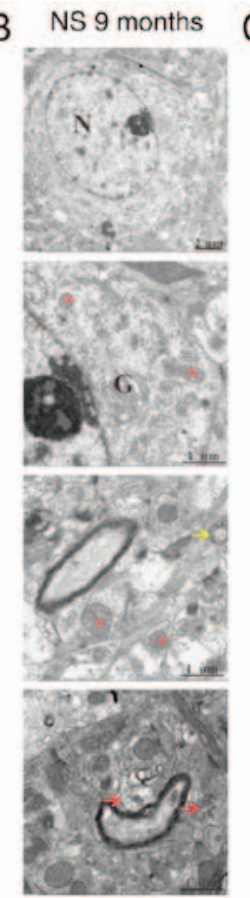

C
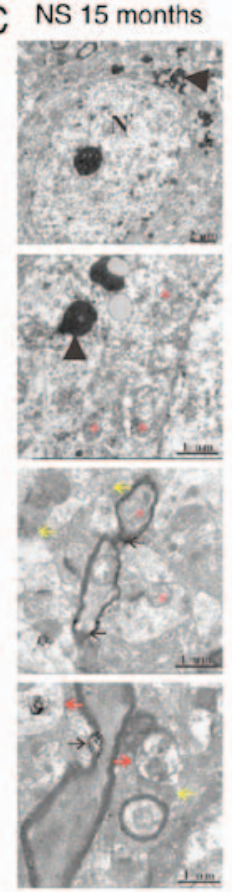
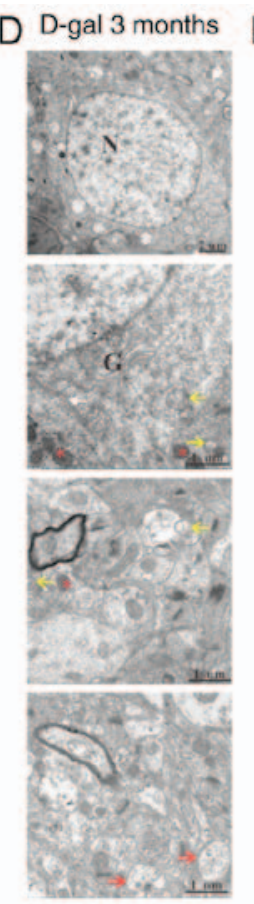

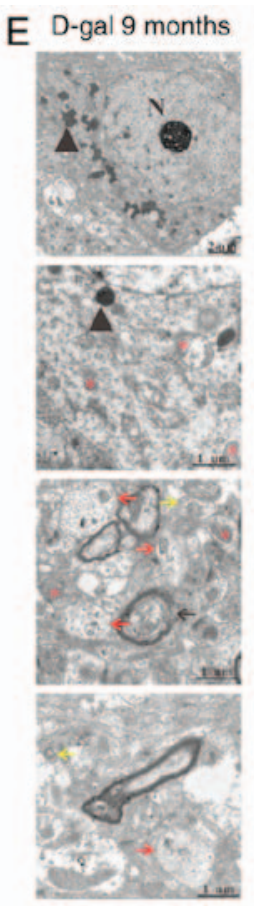

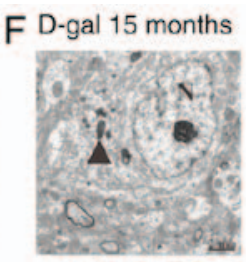

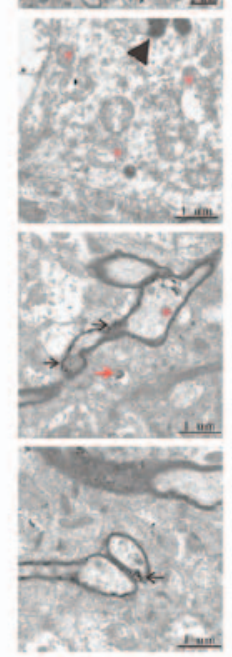

Figure 2. Ultrastructural morphology changes with aging in the NS and D-gal groups. Transmission electron microscopy demonstrated ultrastructural changes in the auditory cortex of the NS and D-gal groups at different ages. Transmission electron microscopy images of the NS groups at (A) 3 , (B) 9 and (C) 15 months, and the D-gal groups at (D) 3, (E) 9 and (F) 15 months. The red asterisks indicate the mitochondria; the black arrows indicate the disrupted myelin; the yellow arrows indicate the autophagosomes; the red arrows indicate the auto-lysosomes; the white arrows indicate the endoplasmic reticulum; and the black arrowheads indicate the lipofuscin. Scale bar, $2 \mathrm{~mm}$ at magnification, x1,700 and $1 \mathrm{~mm}$ at magnification, x5,000. Sections were stained with uranyl acetate and lead citrate. N, nucleus; G, Golgi apparatus; NS, normal saline; D-gal, D-galactose. 

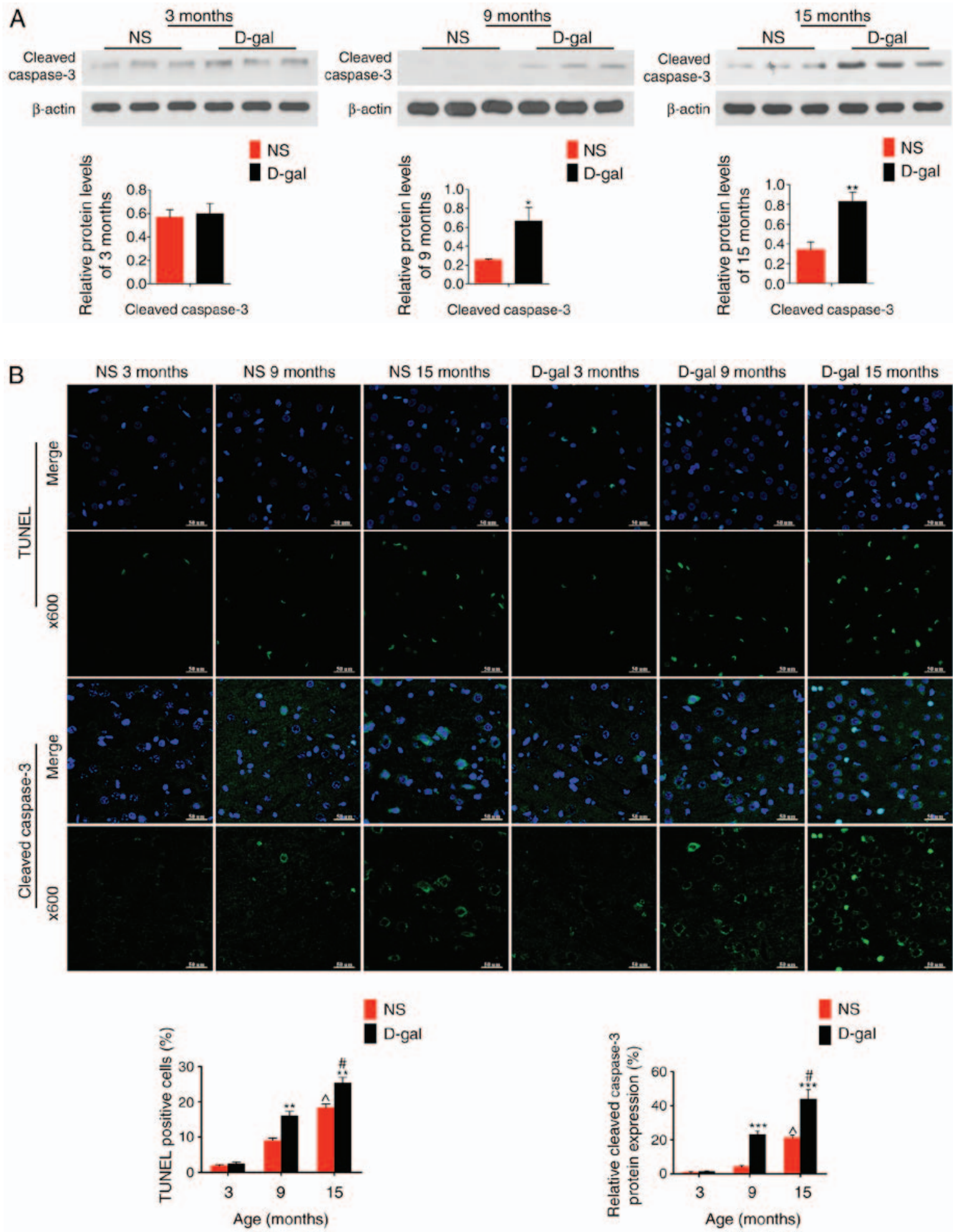

D-gal 9 months D-gal 15 months
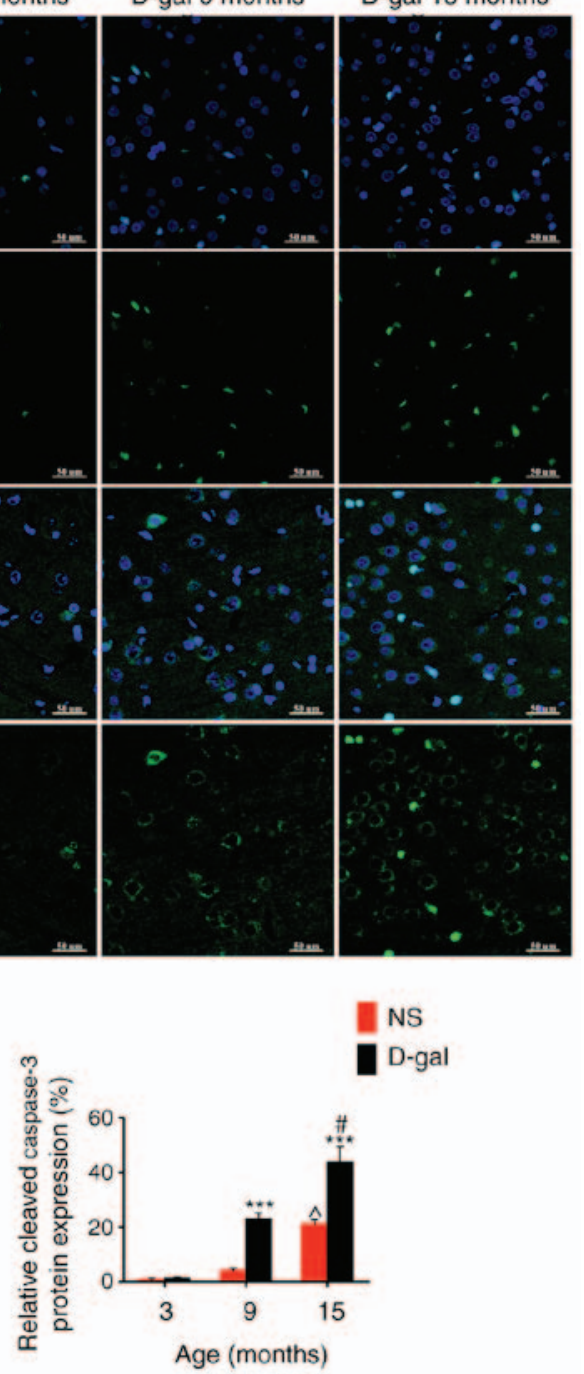

Figure 3. Cell apoptosis level is altered at different ages in the auditory cortex in NS- and D-gal-treated rats. The results of (A) cleaved CASP3 protein expression by western blotting, and (B) immunofluorescence and TUNEL staining were used to reflect the apoptosis level. $\beta$-actin was included as a loading control, and DAPI was used to stain the nuclei. ${ }^{*} \mathrm{P}<0.05,{ }^{* *} \mathrm{P}<0.01$ and ${ }^{* * * *} \mathrm{P}<0.001$ vs. the age-matched NS subgroup; $\wedge \mathrm{P}<0.001$ vs. the 3 -month-old NS subgroup; ${ }^{\text {"}} \mathrm{P}<0.001$ vs. the 3-month-old D-gal subgroup. Data are expressed as mean + standard error of the mean. Scale bar, $50 \mathrm{~mm}$ at magnification, $\mathrm{x} 600$. NS, normal saline; D-gal, D-galactose; CASP3, caspase 3; TUNEL, terminal deoxynucleotidyl-transferase-mediated dUTP nick end labelling.

rats in the 15-month-old NS subgroup and 9-month-old D-gal subgroup were classified as late adults; 15-month-old rats in the D-gal-induced mimetic aging group demonstrated more severe aging than that of other subgroups and should be classified in the old period.
Based on the present results, it was demonstrated that cell apoptosis level increased with aging in the auditory cortex of SD rats, while the level of autophagy showed an increasing trend from young to adult rats and decreased at an old age (Fig. 6). 
A a

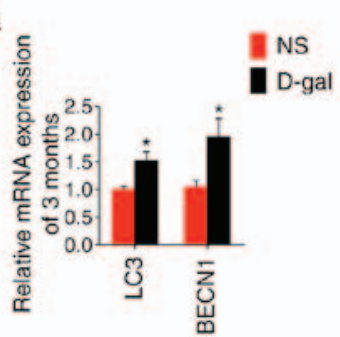

b

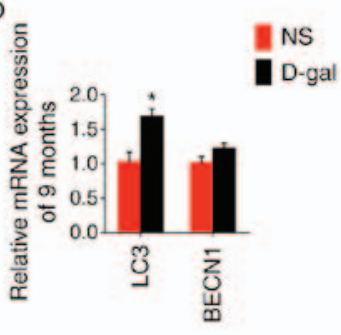

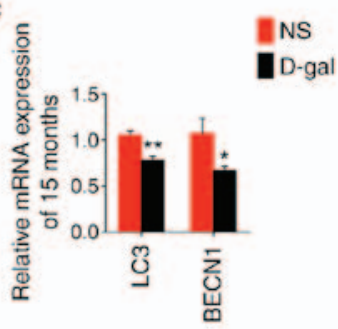

d

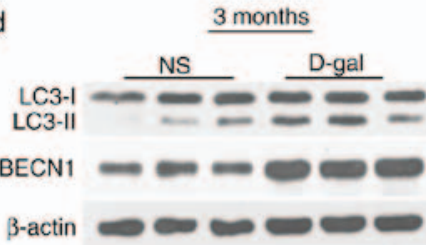

e

9 months

15 months
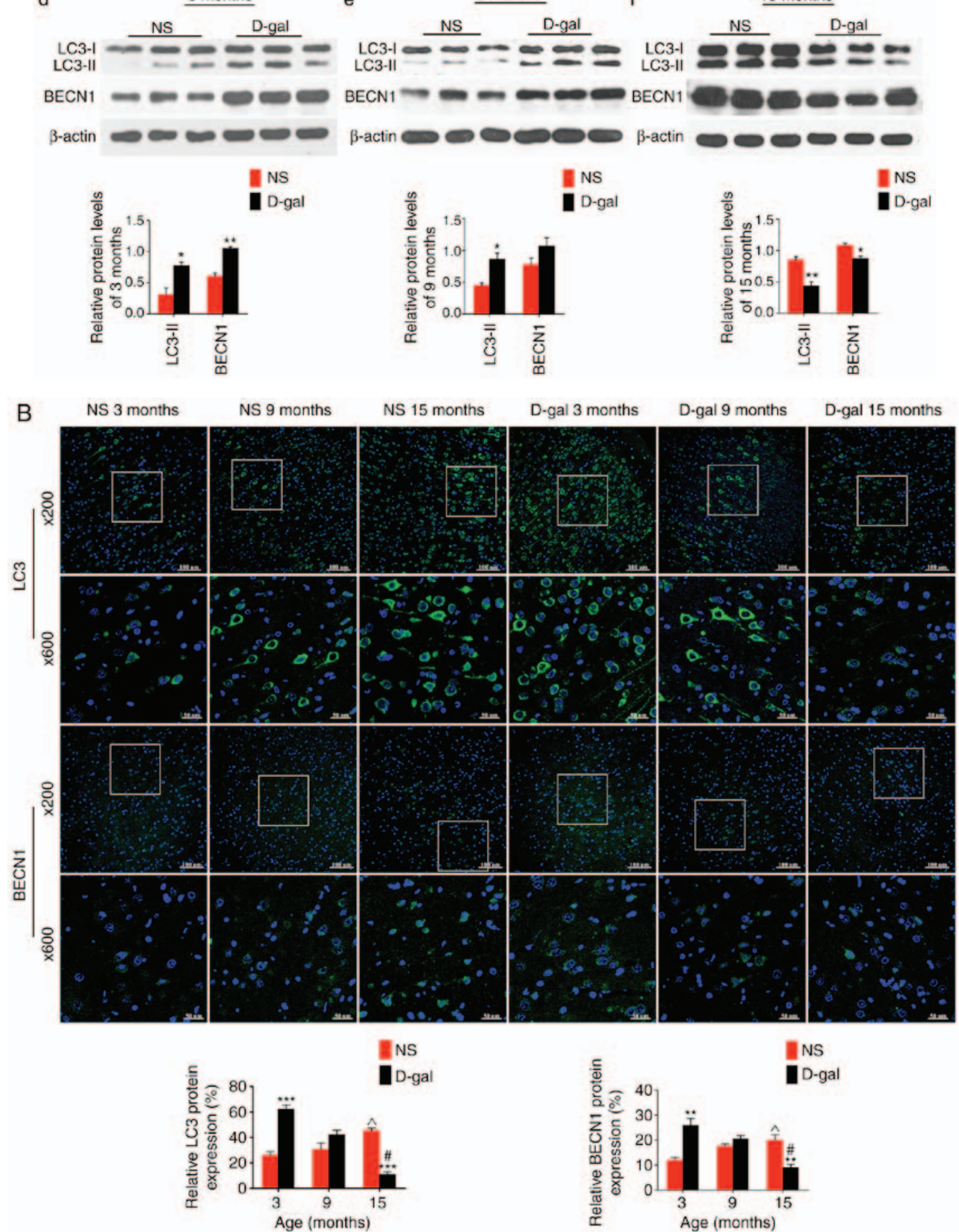

Figure 4. mRNA and protein expression levels of LC3 and BECN1 in the natural aging group and D-gal-induced mimetic aging group. (Aa-c) Reverse transcription-quantitative polymerase chain reaction was used to determine the changes in the mRNA expression levels in the D-gal group compared with those in the age-matched NS group. (Ad-f) Western blot analysis was used to detect the protein expression levels of LC3-II and BECN1. $\beta$-actin was included as a loading control. (B) Changes in LC3 and BECN1 protein expression with aging were demonstrated using immunofluorescence, and the nuclei were stained with DAPI. Data are expressed as mean + standard error of the mean. ${ }^{*} \mathrm{P}<0.05,{ }^{* *} \mathrm{P}<0.01$ and ${ }^{* * *} \mathrm{P}<0.001$ vs. the age-matched NS subgroup; ${ }^{\wedge} \mathrm{P}<0.01$ vs. the 3-month-old NS subgroup; ${ }^{\mathrm{P}} \mathrm{P}<0.001$ vs. the 3-month-old D-gal subgroup. Scale bar, $100 \mathrm{~mm}$ at magnification, $\mathrm{x} 200$ and $50 \mathrm{~mm}$ at magnification, x600. NS, normal saline; D-gal, D-galactose; BECN1, Beclin 1. 


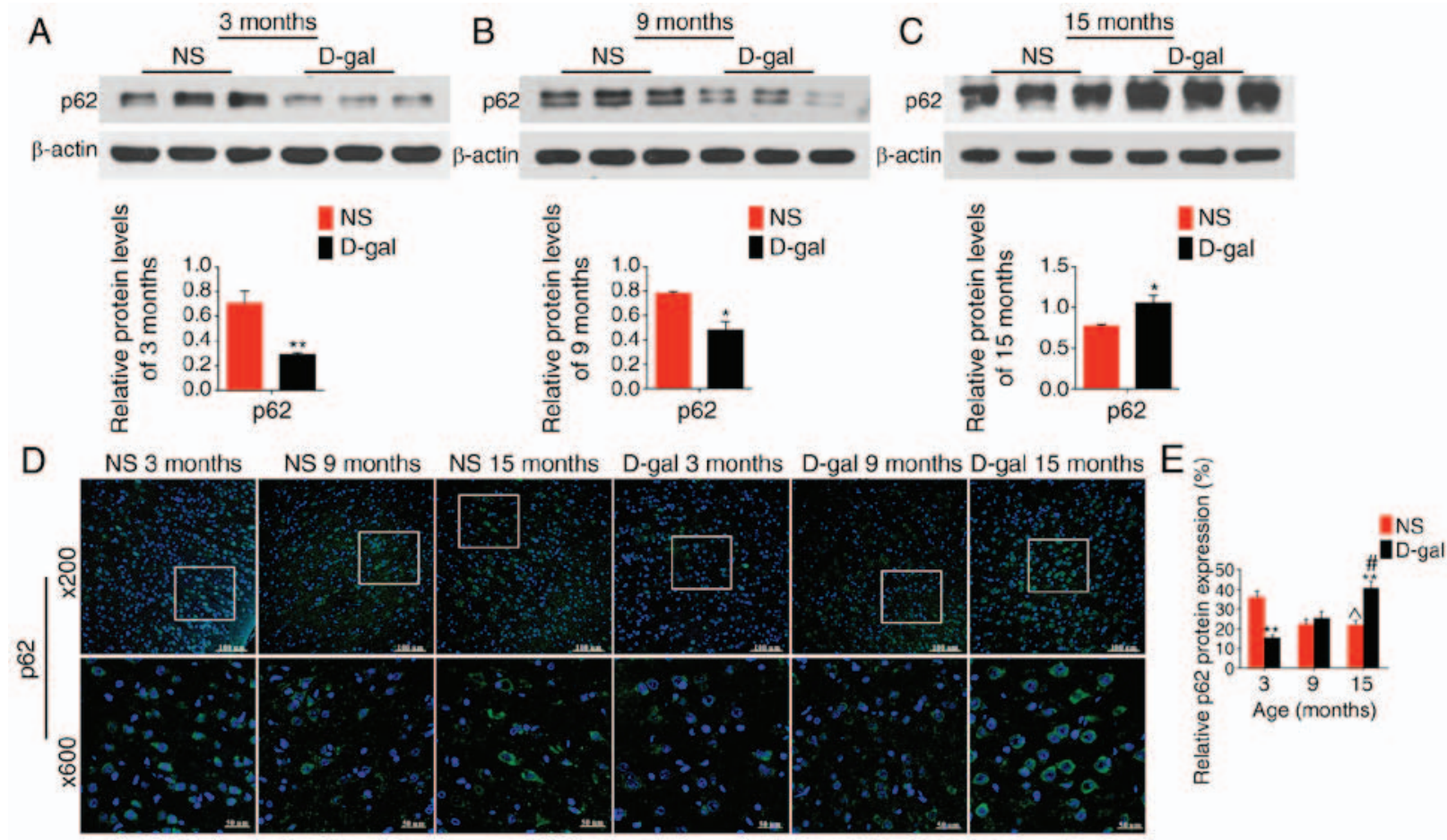

Figure 5. Protein expression levels of p62 in the auditory cortex at different ages in NS- and D-gal-treated rats. Western blot analysis of the NS and D-gal groups at (A) 3, (B) 9 and (C) 15 months. (D) Immunofluorescence assays were used to detect p62 protein expression levels, the nuclei were stained with DAPI and $(\mathrm{E})$ the levels were quantified. $\beta$-actin was included as a loading control. Data are expressed as mean + standard error of the mean. ${ }^{*} \mathrm{P}<0.05$ and ${ }^{* *} \mathrm{P}<0.01 \mathrm{vs}$. the age-matched NS subgroup; ${ }^{\wedge} \mathrm{P}<0.05$ vs. the 3 -month-old NS subgroup; ${ }^{*} \mathrm{P}<0.001$ vs. the 3 -month-old $\mathrm{D}$-gal subgroup. Scale bar, $100 \mathrm{~mm}$ at magnification, x200 and $50 \mathrm{~mm}$ at magnification, x600. NS, normal saline; D-gal, D-galactose.
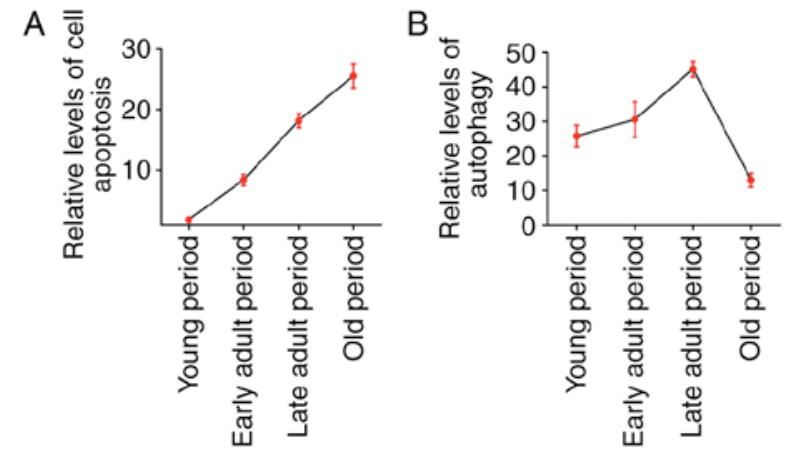

Figure 6. Time-dependent changes in apoptosis and autophagy in the auditory cortex of Sprague Dawley rats. (A) The trend in cell apoptosis with aging was based on the results of terminal deoxynucleotidyl-transferase-mediated dUTP nick end labelling staining. (B) The trend in autophagy changes with aging was based on the results of the immunofluorescence analysis of the autophagy marker, LC3. The 3-, 9- and 15-month-old rats in the normal saline groups and the 15-month-old rats in D-galactose group represent the young period, early adult period, late adult period and old period, respectively.

$D$-gal treatment initially increases anti-apoptotic proteins, including $B C L 2$ and $B C L-x L$, and then impairs them. Previous research has suggested that autophagy has an anti-apoptotic effect in injured spinal cord neurons (46). To investigate the role of autophagy in the present study, in addition to the detection of apoptosis level by TUNEL analysis and cleaved CASP3, the changes in the BCL2 family proteins (BCL2, BCL-xL and BAX) were also assessed. mRNA and protein expression levels of anti-apoptotic proteins, BCL2 and BCL-xL, as well as the ratio of BCL2/BAX and
BCL-xL/BAX were significantly increased at 3 months in the mimetic aging groups but decreased at 15 months compared with those in the age-matched NS control groups. However, the mRNA and protein levels of pro-apoptotic protein, BAX, demonstrated no significant changes at 3 months in the mimetic aging groups, but increased significantly at 9 and 15 months compared with those in the age-matched NS control groups (Fig. 7A). The immunofluorescence images demonstrated that BCL2 was increased from 3 to 15 months in the NS group, while it decreased with aging in the D-gal group (Fig. 7B).

$D$-gal treatment induces activation of the AMPK-mTOR-ULK1 signaling pathway in the auditory cortex. To investigate the hypotheses regarding the activation and inhibition of autophagy, the protein expression levels of the AMPK-mTOR-ULK1 signaling pathway in the auditory cortex were detected using western blotting. Representative western blots demonstrated that the expression of p-AMPK was significantly increased in a compensatory manner at 3 months, but significantly decreased at 9 and 15 months in the D-gal groups compared with its expression in the age-matched NS group. However, p-mTOR and p-ULK1 demonstrated an inverse trend with p-AMPK. Additionally, the autophagy-related protein Atg13 was significantly increased at 3 and 9 months in the D-gal group compared with the levels in the age-matched NS group (Fig. 8), which was consistent with the expression results for LC3-II and BECN1.

The 4EBP1 phosphorylation level was also investigated. As demonstrated in Fig. 9, the expression level of p-4EBP1 was 

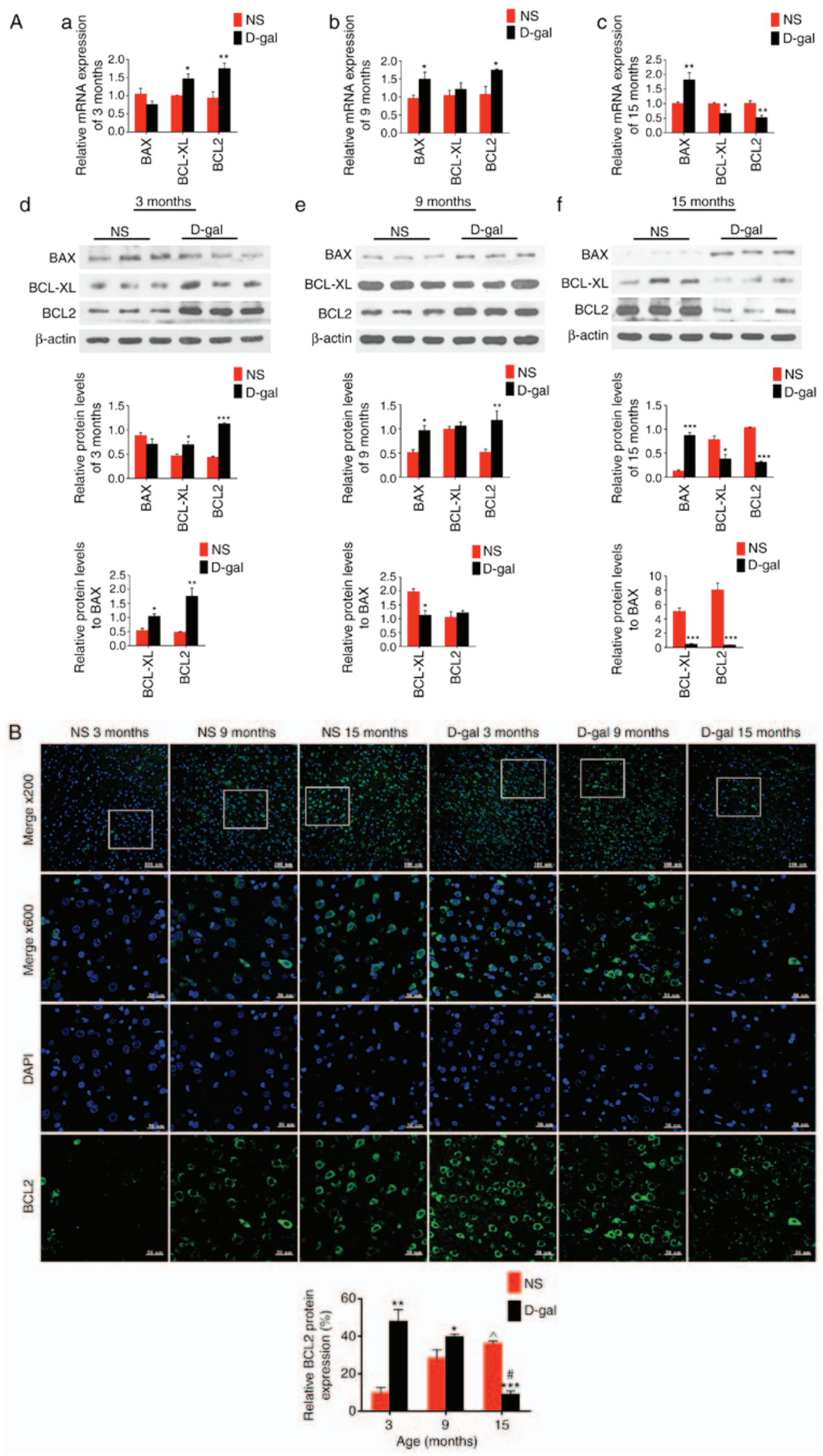

Figure 7. mRNA and protein expression levels of BCL2, BCL-xL and BAX in the NS natural aging group and D-gal-induced mimetic aging group. (Aa-c) Reverse transcription-quantitative polymerase chain reaction and (Ad-f) western blotting were used to study the mRNA and protein expression levels in the D-gal group and NS group, respectively. $\beta$-actin was used as a loading control. (B) Visualization of changes of BCL2 protein expression with aging was demonstrated using immunofluorescence, and the nuclei were stained with DAPI. Data are expressed as mean \pm standard error of the mean. ${ }^{*} \mathrm{P}<0.05,{ }^{, * *} \mathrm{P}<0.01$ and ${ }^{* * *} \mathrm{P}<0.001$ vs. the age-matched NS subgroup; ${ }^{\wedge} \mathrm{P}<0.01$ vs. the 3 -month-old NS subgroup; ${ }^{*} \mathrm{P}<0.001$ vs. the 3 -month-old $\mathrm{D}$-gal subgroup. Scale bar, $100 \mathrm{~mm}$ at magnification, x200 and $50 \mathrm{~mm}$ at magnification, x600. NS, normal saline; D-gal, D-galactose; BCL2, B-cell lymphoma 2; BCL-xL, B-cell lymphoma-extra large; BAX, B-cell lymphoma 2-associated X protein. 
A
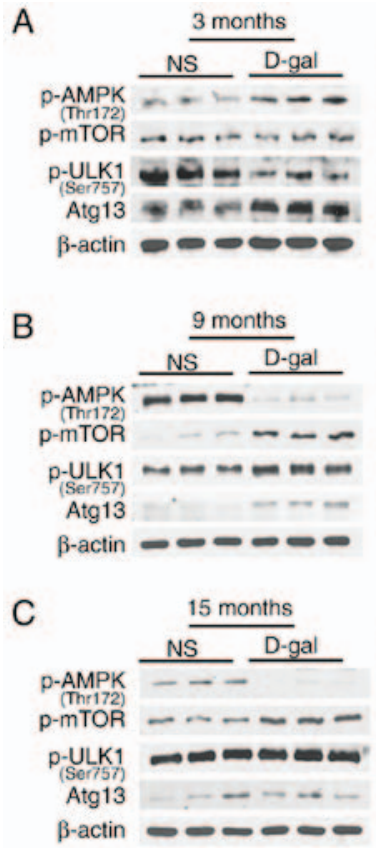
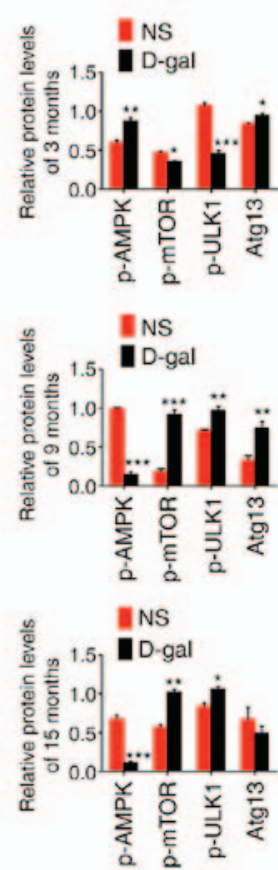

Figure 8. Effects of D-gal treatment on the AMPK-mTOR-ULK1 signaling pathway in the auditory cortex. Western blotting was used to detect the protein expression levels of the AMPK-mTOR-ULK1 signaling pathway in the (A) 3-, (B) 9- and (C) 15-month-old NS and D-gal groups. $\beta$-actin was included as a loading control. Data are expressed as mean + standard error of the mean. ${ }^{*} \mathrm{P}<0.05,{ }^{* *} \mathrm{P}<0.01$ and ${ }^{* * *} \mathrm{P}<0.001$ vs. the age-matched NS subgroups. NS, normal saline; D-gal, D-galactose; AMPK, 5' AMP-activated protein kinase; mTOR, mechanistic target of rapamycin; $p$, phosphorylated Atg, autophagy-related protein.
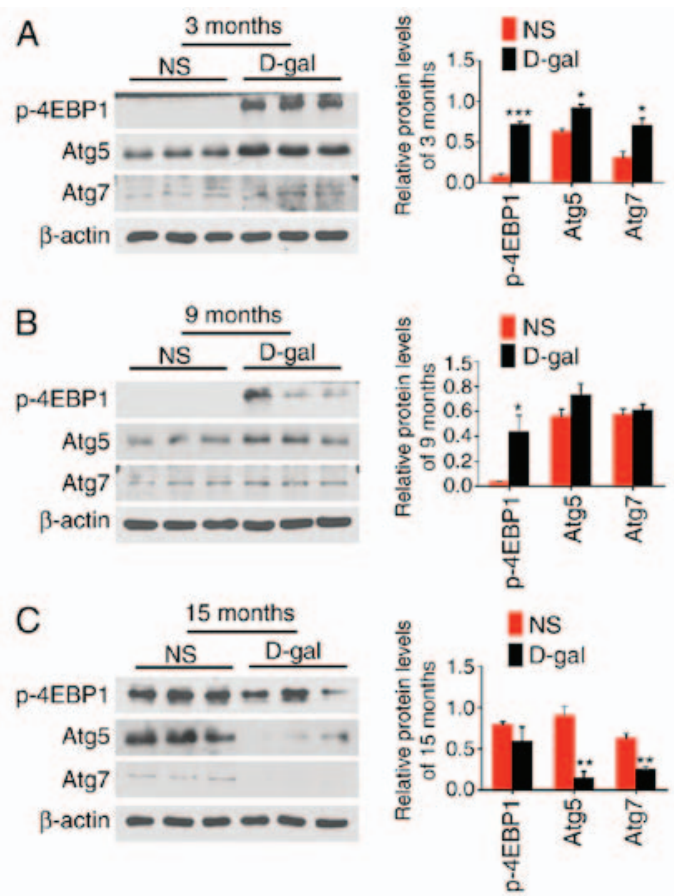

Figure 9. Changes to the p-4EBP1 protein expression levels in the auditory cortex with aging in the NS and D-gal-induced mimetic aging rats. The effects of D-gal treatment on the expression of p-4EBP1 in the (A) 3(B) 9- and (C) 15-month-old NS and D-gal groups as determined by western blotting. There was no obvious correlation between p-4EBP1 and autophagy-related proteins. $\beta$-actin was used as a loading control. Data are expressed as mean + standard error of the mean. ${ }^{*} \mathrm{P}<0.05$ and ${ }^{* *} \mathrm{P}<0.01$ vs. the age-matched NS subgroups. NS, normal saline; D-gal, D-galactose; $\mathrm{p}$, phosphorylated; Atg, autophagy-related protein.

significantly increased at 3 and 9 months in the D-gal groups compared with that in the age-matched NS groups. However, the autophagy-related proteins Atg5 and Atg7 were significantly increased at 3 months, while significantly decreased at 15 months in D-gal groups compared with the levels in the age-matched NS group. These results suggest that there is no obvious relevance between 4EBP1 activity and autophagy regulation. Based on these findings, it was hypothesized that p-4EBP1 was not involved in the inhibition of autophagy in the present model. Additionally, the potential relationships among AMPK, mTOR, p-ULK1 (Ser 757), autophagy and apoptosis in the present model are presented in Fig. 10.

\section{Discussion}

Autophagy is a cellular catabolic process that is essential for survival, differentiation, development and homeostasis (47). A large body of evidence indicates that autophagy declines with aging $(48,49)$. However, age-related increases of autophagy in rat nucleus pulposus and pancreatic islet cells have also been reported $(50,51)$. Recent research has suggested that autophagy increases from the perinatal period to adulthood and then declines after the age of 12 months in the inner ear of mice (52). However, no studies have examined autophagy in the auditory cortex and the changes with age. In the present study, 3-, 9- and 15-month-old rats in the natural aging and D-gal induced mimetic aging groups were observed. The present results demonstrated that autophagy-related proteins, LC3 and BECN1, were increased from 3 months to 15 months in the natural aging group and decreased at 15 months in the mimetic aging group, while the degradation of p62 demonstrated an inverse trend. These results suggest that autophagy level is time-dependent and increases from young to adult rats and decreases at an old age in the auditory cortex of SD rats.

Ultrastructural morphology of neurons and cell apoptosis level in the mimetic aging group demonstrated no significant differences with those of the age-matched NS group, while the autophagy level increased. However, at 15 months, it was observed that autophagy in the mimetic aging group was significantly decreased compared to that in the age-matched natural aging group, and neuron degeneration and cell apoptosis were more severe than that in the NS group, as expected. It was concluded that autophagy serves a protective role in the degeneration of the auditory cortex, and a compensatory increase in autophagy at 3 months protected the auditory cortex from D-gal-induced premature senescence. Deficient or impaired autophagy lost its protective function, leading to the acceleration of apoptosis and finally senescence.

The protective role of autophagy was further demonstrated by investigating anti-apoptotic protein levels of BCL2 and BCL-xL in the present study. It was demonstrated that autophagy had a similar trend to that of BCL2 and BCL-xL in natural aging and mimetic aging rats, and it was concluded that autophagy cooperated with BCL2 and BCL-xL to exert anti-apoptotic effects in the present rat model. However, BCL2 and BCL-xL have been reported to suppress autophagy by binding BECN1 directly via its BH3 domain, preventing activation of autophagy $(53,54)$; however, relevant evidence showing that autophagy was inhibited 


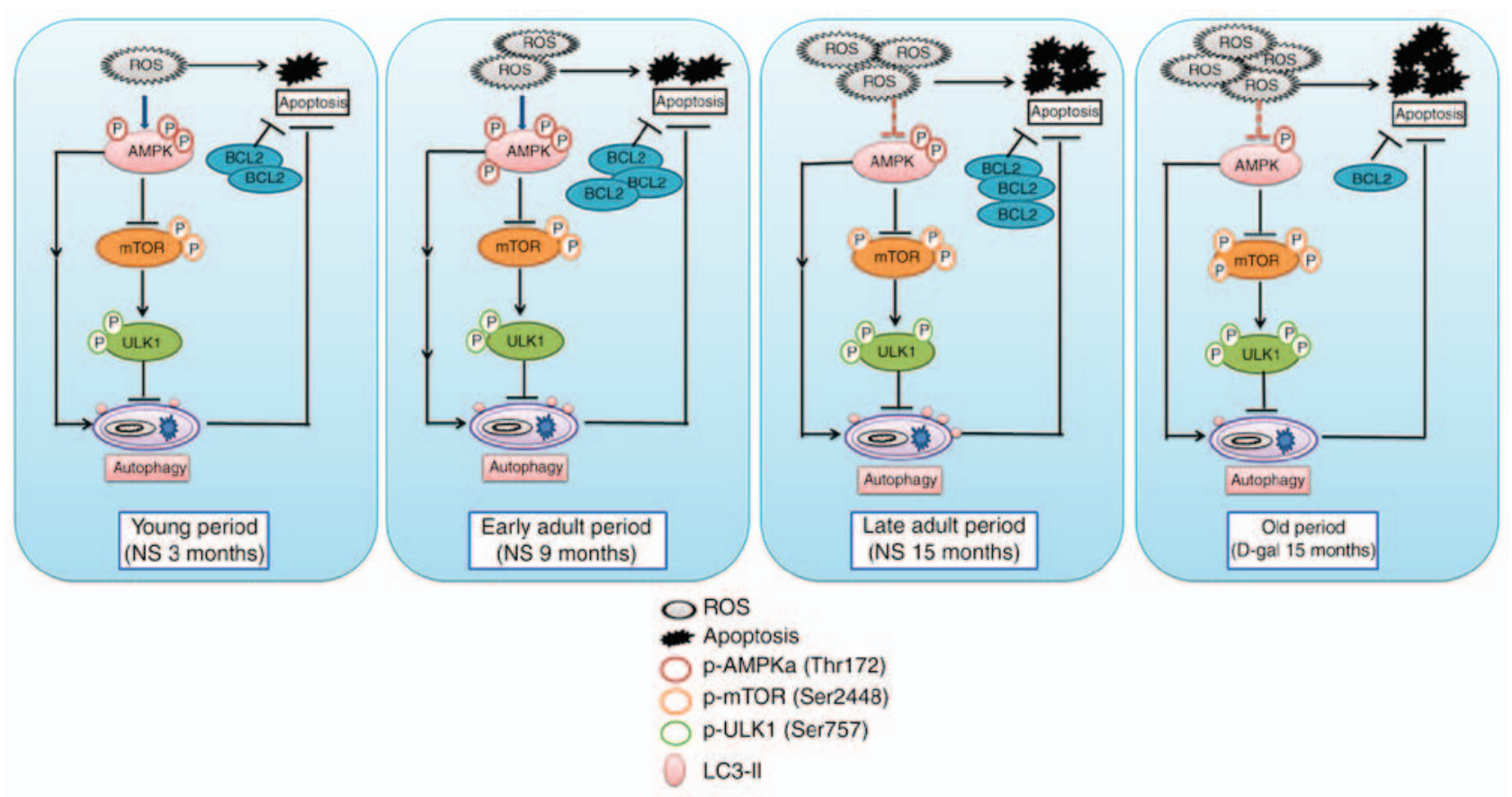

Figure 10. Schematic of the interrelationships among p-AMPK, p-mTOR, p-ULK1, autophagy and apoptosis. Accumulated ROS results in the phosphorylation and activation of AMPK. Activated AMPK suppresses mTOR, thereby activating autophagy and exerting an anti-apoptotic function. Additionally, AMPK may directly activate autophagy through other mechanisms. However, excessive ROS and metabolites lead to impairment of AMPK activity, and increased activity of mTOR phosphorylates ULK1 at Ser 757, leading to the suppression of autophagy initiation. Autophagy and BCL2 cooperate against apoptosis and delay the process of senescence. AMPK, 5' AMP-activated protein kinase; mTOR, mechanistic target of rapamycin; p, phosphorylated; ROS, reactive oxygen species; BCL2, B-cell lymphoma 2; NS, normal saline; D-gal, D-galactose.

by BCL2 and BCL-xL was not identified in the present study. In addition, Pattingre et al (55) demonstrated that BCL2 interacts with BECN1 to maintain autophagy at levels that are compatible with cell survival rather than cell death, and Al-Shenawy (56) found a positive correlation between BECN1 and BCL-XL/BCL2 expression in studies on autophagy-related marker, BECN1, and its relationship with apoptotic markers in chronic hepatitis and hepatocellular carcinoma. Hence, the present study assumed that BCL2 and BCL-xL acted as anti-apoptotic proteins rather than inhibitors of autophagy.

Activation of AMPK was significantly increased in the mimetic aging group at 3 months, and p-mTOR and p-ULK1 (Ser 757) levels decreased, which could account for the rise of autophagy at 3 months in the mimetic aging group. However, an accordant decrease of autophagy at 9 months in the D-gal group was not identified, while AMPK activity was notably decreased and p-mTOR and p-ULK1 were increased. It was hypothesized that the loss of activation by AMPK and increased inhibition by mTOR failed to suppress autophagy activity until 15 months. As a major inhibitor of autophagy, high mTOR activity prevents ULK1 activation by phosphorylating it at Ser 757, disrupting the interaction between ULK1 and AMPK, and finally suppressing autophagy (20). Previous research has indicated that increased 4EBP1 activity results in the impairment of autophagy in $\mathrm{H}_{2} \mathrm{O}_{2}$-induced premature senescence in auditory cells (25). However, by detecting 4EBP1 activity and autophagy-related proteins, Atg5 and Atg7, the present study demonstrated that 4EBP1 phosphorylation was increased at 3 and 9 months in the D-gal group compared with that in the age-matched NS group, and no significant difference was observed at 15 months. Atg5 and Atg7 were increased at
3 months and decreased at 15 months, indicating that 4EBP1 activity may be not involved in the regulation of autophagy in the present model. Additionally, the present results revealed that 4EBP1 phosphorylation may be independent of mTOR, which is consistent with previous studies $(25,57)$. We hypothesized that 4EBP1 may have different functions in tissues and cells, and the specific role of 4EBP1 in regulating autophagy and its related mechanisms require further study. The present results demonstrated that AMPK and mTOR, as well as ULK1, are involved in the regulation of autophagy; however, the relationships between AMPK and autophagy, and mTOR and autophagy, need to be clarified. Research has demonstrated that death-associated protein 1 is a novel mTOR substrate and has an inhibitory role in autophagy (58).

In summary, by studying autophagy and apoptosis, as well as the AMPK-mTOR-ULK1 signaling pathway, in naturally aging rats and mimetic aging rats at 3,9 and 15 months, the present study demonstrated that autophagy increased in a time-dependent manner (from young to adult) and then decreased at old age. Furthermore, autophagy acts in a compensatory manner to block apoptosis and maintain homeostasis in the body, whereas the loss of AMPK activity and increased inhibition of mTOR signaling impaired autophagy. This may be one mechanism underlying the degeneration of the auditory cortex and may be partially responsible for the pathogenesis of ARHL. Although the molecular mechanisms involved in ARHL have yet to be fully elucidated, the present results provide important insights into the role of autophagy during D-gal-induced premature senescence of the auditory cortex. Based on these results, the activation of AMPK and the suppression of mTOR signaling may upregulate autophagy, which may delay the neurodegenerative 
process. However, further studies are required to elucidate the mechanisms of AMPK-mTOR-ULK1 signaling and other pathways that regulate autophagy, as well as the connections between autophagy and apoptosis, to determine how senescence and autophagy affect auditory cortex function and contribute to the pathology of hearing impairments.

\section{Acknowledgements}

The present study was supported by the National Natural Science Foundation of China (grant no. 81230021) and the Major State Basic Research Development Program of China (973 program; grant no. 2011CB504504).

\section{Competing interests}

The authors declare that they have no competing interests.

\section{References}

1. Gao F, Wang G, Ma W, Ren F, Li M, Dong Y, Liu C, Liu B, Bai X, Zhao B and Edden RA: Decreased auditory GABA+ concentrations in presbycusis demonstrated by edited magnetic resonance spectroscopy. Neuroimage 106: 311-316, 2015.

2. Yamasoba T, Lin FR, Someya S, Kashio A, Sakamoto T and Kondo K: Current concepts in age-related hearing loss: Epidemiology and mechanistic pathways. Hear Res 303: 30-38, 2013.

3. Menardo J, Tang Y, Ladrech S, Lenoir M, Casas F, Michel C, Bourien J, Ruel J, Rebillard G, Maurice T, et al: Oxidative stress, inflammation, and autophagic stress as the key mechanisms of premature age-related hearing loss in SAMP8 mouse Cochlea. Antioxid Redox Signal 16: 263-274, 2012.

4. Mazelová J, Popelar J and Syka J: Auditory function in presbycusis: Peripheral vs. central changes. Exp Gerontol 38: 87-94, 2003.

5. Gates GA, Feeney MP and Mills D: Cross-sectional age-changes of hearing in the elderly. Ear Hear 29: 865-874, 2008.

6. Ho SC, Liu JH and Wu RY: Establishment of the mimetic aging effect in mice caused by D-galactose. Biogerontology 4: 15-18, 2003.

7. Lee J, Cho JY and Kim WK: Anti-inflammation effect of Exercise and Korean red ginseng in aging model rats with diet-induced atherosclerosis. Nut Res Pract 8: 284-291, 2014.

8. Ahangarpour A, Lamoochi Z, Fathi Moghaddam H and Mansouri SM: Effects of Portulaca oleracea ethanolic extract on reproductive system of aging female mice. Int $\mathrm{J}$ Reprod Biomed 14: 205-212, 2016.

9. Liao CH, Chen BH, Chiang HS, Chen $\mathrm{CW}$, Chen MF, Ke CC, Wang YY, Lin WN, Wang CC and Lin YH: Optimizing a Male reproductive aging mouse model by $\mathrm{D}$-galactose injection. Int $\mathrm{J}$ Mol Sci 17: E98, 2016.

10. Gao J, Zhou R, You X, Luo F, He H, Chang X, Zhu L, Ding $X$ and Yan T: Salidroside suppresses inflammation in a D-galactose-induced rat model of Alzheimer's disease via SIRT1/NF-kappaB pathway. Metab Brain Dis 31: 771-778, 2016.

11. Zhong Y, Hu YJ, Chen B, Peng W, Sun Y, Yang Y, Zhao XY, Fan GR, Huang $X$ and Kong WJ: Mitochondrial transcription factor A overexpression and base excision repair deficiency in the inner ear of rats with D-galactose-induced aging. FEBS J 278 : 2500-2510, 2011.

12. Wu L, Sun Y, Hu YJ, Yang Y, Yao LL, Zhou XX, Wang H, Zhang R, Huang $X$ and Kong WJ: Increased p66Shc in the inner ear of D-galactose-induced aging mice with accumulation of mitochondrial DNA 3873-bp deletion: p66Shc and mtDNA damage in the inner ear during aging. PLoS One 7: e50483, 2012.

13. Klionsky DJ and Emr SD: Autophagy as a regulated pathway of cellular degradation. Science 290: 1717-1721, 2000.

14. Jia G and Sowers JR: Autophagy: A housekeeper in cardiorenal metabolic health and disease. Biochim Biophys Acta 1852: 219-224, 2015.
15. Kim I and Lemasters JJ: Mitochondrial degradation by autophagy (mitophagy) in GFP-LC3 transgenic hepatocytes during nutrient deprivation. Am J Physiol Cell Physiol 300: C308-C317, 2011.

16. Lee JA: Neuronal autophagy: A housekeeper or a fighter in neuronal cell survival? Exp Neurobiol 21: 1-8, 2012.

17. Shen HM and Codogno P: Autophagy is a survival force via suppression of necrotic cell death. Exp Cell Res 318: 1304-1308, 2012.

18. Loos B, Engelbrecht AM, Lockshin RA, Klionsky DJ and Zakeri Z: The variability of autophagy and cell death susceptibility: Unanswered questions. Autophagy 9: 1270-1285, 2013.

19. Shang L and Wang X: AMPK and mTOR coordinate the regulation of Ulk1 and mammalian autophagy initiation. Autophagy 7: 924-926, 2011.

20. Kim J, Kundu M, Viollet B and Guan KL: AMPK and mTOR regulate autophagy through direct phosphorylation of Ulk1. Nat Cell Biol 13: 132-141, 2011.

21. Hosokawa N, Hara T, Kaizuka T, Kishi C, Takamura A Miura Y, Iemura S, Natsume T, Takehana K, Yamada N, et al: Nutrient-dependent mTORC1 association with the ULK1-Atg13FIP200 complex required for autophagy. Mol Biol Cell 20: 1981-1991, 2009.

22. Jung CH, Ro SH, Cao J, Otto NM and Kim DH: mTOR regulation of autophagy. FEBS Lett 584: 1287-1295, 2010.

23. Mihaylova MM and Shaw RJ: The AMPK signalling pathway coordinates cell growth, autophagy and metabolism. Nat Cell Biol 13: 1016-1023, 2011.

24. Perl A: Oxidative stress in the pathology and treatment of systemic lupus erythematosus. Nat Rev Rheumatol 9: 674-686, 2013.

25. Tsuchihashi NA, Hayashi K, Dan K, Goto F, Nomura Y, Fujioka M, Kanzaki S, Komune S and Ogawa K: Autophagy through 4EBP1 and AMPK regulates oxidative stress-induced premature senescence in auditory cells. Oncotarget 6: 3644-3655, 2015.

26. Salminen A and Kaarniranta K: AMP-activated protein kinase (AMPK) controls the aging process via an integrated signaling network. Ageing Res Rev 11: 230-241, 2012.

27. Johnson SC, Rabinovitch PS and Kaeberlein M: mTOR is a key modulator of ageing and age-related disease. Nature 493: 338-345, 2013.

28. Crino PB: The mTOR signalling cascade: Paving new roads to cure neurological disease. Nat Rev Neurol 12: 379-392, 2016.

29. Song JX, Sun YR, Peluso I, Zeng Y, Yu X, Lu JH, Xu Z, Wang MZ, Liu LF, Huang YY, et al: A novel curcumin analog binds to and activates TFEB in vitro and in vivo independent of MTOR inhibition. Autophagy 12: 1372-1389, 2016.

30. Meijer AJ and Codogno P: AMP-activated protein kinase and autophagy. Autophagy 3: 238-240, 2007.

31. Huang YH, Al-Aidaroos AQ, Yuen HF, Zhang SD, Shen HM, Rozycka E, McCrudden CM, Tergaonkar V, Gupta A, Lin YB, et al: A role of autophagy in PTP4A3-driven cancer progression. Autophagy 10: 1787-1800, 2014.

32. Lee E, Koo Y, Ng A, Wei Y, Luby-Phelps K, Juraszek A, Xavier RJ, Cleaver O, Levine B and Amatruda JF: Autophagy is essential for cardiac morphogenesis during vertebrate development. Autophagy 10: 572-587, 2014.

33. Komatsu M, Waguri S, Chiba T, Murata S, Iwata J, Tanida I, Ueno T, Koike M, Uchiyama Y, Kominami E and Tanaka K: Loss of autophagy in the central nervous system causes neurodegeneration in mice. Nature 441: 880-884, 2006.

34. Gianchecchi E, Delfino DV and Fierabracci A: Recent insights on the putative role of autophagy in autoimmune diseases. Autoimmun Rev 13: 231-241, 2014.

35. Munch D, Rodriguez E, Bressendorff S, Park OK, Hofius D and Petersen M: Autophagy deficiency leads to accumulation of ubiquitinated proteins, ER stress, and cell death in Arabidopsis. Autophagy 10: 1579-1587, 2014.

36. Pattingre S, Bauvy C, Carpentier S, Levade T, Levine B and Codogno P: Role of JNK1-dependent Bcl-2 phosphorylation in ceramide-induced macroautophagy. J Biol Chem 284: 2719-2728, 2009.

37. Ito $\mathrm{H}$, Daido $\mathrm{S}$, Kanzawa $\mathrm{T}$, Kondo $\mathrm{S}$ and Kondo $\mathrm{Y}$ : Radiation-induced autophagy is associated with LC3 and its inhibition sensitizes malignant glioma cells. Int J Oncol 26: 1401-1410, 2005.

38. Saita S, Shirane M and Nakayama KI: Selective escape of proteins from the mitochondria during mitophagy. Nat Commun 4: 1410, 2013.

39. National Research Council Committee for the Update of the Guide for the $\mathrm{C}$ and Use of Laboratory A: The National Academies Collection: Reports funded by National Institutes of Health. In: Guide for the Care and Use of Laboratory Animals. th (ed). National Academies Press (US), National Academy of Sciences, Washington (DC), 2011. 
40. Nicklas JA, Brooks EM, Hunter TC, Single R and Branda RF: Development of a quantitative PCR (TaqMan) assay for relative mitochondrial DNA copy number and the common mitochondrial DNA deletion in the rat. Environ Mol Mutagen 44: 313-320, 2004.

41. Livak KJ and Schmittgen TD: Analysis of relative gene expression data using real-time quantitative PCR and the $2^{-\Delta \Delta C \mathrm{~T}}$ method. Methods 25: 402-408, 2001.

42. Edris W, Burgett B, Stine OC and Filburn CR: Detection and quantitation by competitive PCR of an age-associated increase in a $4.8-\mathrm{kb}$ deletion in rat mitochondrial DNA. Mutat Res 316: 69-78, 1994.

43. Bai U, Seidman MD, Hinojosa R and Quirk WS: Mitochondrial DNA deletions associated with aging and possibly presbycusis: A human archival temporal bone study. Am J Otol 18: 449-453, 1997.

44. Chen B, Zhong Y, Peng W, Sun Y and Kong WJ: Age-related changes in the central auditory system: Comparison of D-galactose-induced aging rats and naturally aging rats. Brain Res 1344: 43-53, 2010.

45. Andreollo NA, Santos EF, Araújo MR and Lopes LR: Rat's age versus human's age: What is the relationship? Arq Bras Cir Dig 25: 49-51, 2012 (In English, Portuguese)

46. Wang ZY, Lin JH, Muharram A and Liu WG: Beclin-1-mediated autophagy protects spinal cord neurons against mechanical injury-induced apoptosis. Apoptosis 19: 933-945, 2014.

47. Levine B and Kroemer G: Autophagy in the pathogenesis of disease. Cell 132: 27-42, 2008.

48. Cuervo AM, Bergamini E, Brunk UT, Dröge W, Ffrench M and Terman A: Autophagy and aging: The importance of maintaining ‘clean' cells. Autophagy 1: 131-140, 2005.

49. Ott C, König J, Höhn A, Jung T and Grune T: Macroautophagy is impaired in old murine brain tissue as well as in senescent human fibroblasts. Redox Biol 10: 266-273, 2016.
50. Ye W, Xu K, Huang D, Liang A, Peng Y, Zhu W and Li C: Age-related increases of macroautophagy and chaperone-mediated autophagy in rat nucleus pulposus. Connect Tissue Res 52: 472-478, 2011.

51. Wang S, Sun QQ, Xiang B and Li XJ: Pancreatic islet cell autophagy during aging in rats. Clin Invest Med 36: E72-E80, 2013.

52. de Iriarte Rodríguez R, Pulido S, Rodríguez-de la Rosa L, Magariños M and Varela-Nieto I: Age-regulated function of autophagy in the mouse inner ear. Hear Res 330: 39-50, 2015.

53. Decuypere JP, Parys JB and Bultynck G: Regulation of the autophagic bcl-2/beclin 1 interaction. Cells 1: 284-312, 2012.

54. Levine B, Sinha SC and Kroemer G: Bcl-2 family members: Dual regulators of apoptosis and autophagy. Autophagy 4: 600-606, 2008.

55. Pattingre S, Tassa A, Qu X, Garuti R, Liang XH, Mizushima N, Packer M, Schneider MD and Levine B: Bcl-2 antiapoptotic proteins inhibit Beclin 1-dependent autophagy. Cell 122: 927-939, 2005.

56. Al-Shenawy HA: Expression of Beclin-1, an autophagy-related marker, in chronic hepatitis and hepatocellular carcinoma and its relation with apoptotic markers. APMIS 124: 229-237, 2016.

57. Chao SK, Lin J, Brouwer-Visser J, Smith AB, III, Horwitz SB and McDaid HM: Resistance to discodermolide, a microtubule-stabilizing agent and senescence inducer, is 4E-BP1-dependent. Proc Natl Acad Sci U SA 108: 391-396, 2011.

58. Koren I, Reem E and Kimchi A: DAP1, a novel substrate of mTOR, negatively regulates autophagy. Curr Biol 20: 1093-1098, 2010 .

This work is licensed under a Creative Commons Attribution-NonCommercial-NoDerivatives 4.0 International (CC BY-NC-ND 4.0) License. 